\title{
Reflection holograms stored in an environment-friendly photopolymer
}

Manuel G. Ramírez, Daniel Sirvent, Manuel Ortuño, Víctor Navarro-Fuster, Francisco J. Martínez, et al.

Manuel G. Ramírez, Daniel Sirvent, Manuel Ortuño, Víctor Navarro-Fuster, Francisco J. Martínez, Augusto Beléndez, Inmaculada Pascual Villalobos, "Reflection holograms stored in an environment-friendly photopolymer," Proc. SPIE 11030, Holography: Advances and Modern Trends VI, 110300E (14 May 2019); doi: $10.1117 / 12.2521439$

SPIE. Event: SPIE Optics + Optoelectronics, 2019, Prague, Czech Republic 


\title{
Reflection holograms stored in an Environment-Friendly Photopolymer
}

\author{
Manuel G. Ramírez a, , Dani Sirvent ${ }^{\mathrm{a}}$, Manuel Ortuño ${ }^{\mathrm{b}}$, Víctor Navarro-Fuster ${ }^{\mathrm{b}}$, Francisco J. \\ Martínez $^{\mathrm{b}}$, Augusto Beléndez ${ }^{\mathrm{b}}$ and Inmaculada Pascual ${ }^{\mathrm{b}}$ \\ ${ }^{a}$ Departamento de Física, Ingeniería de Sistemas y Teoría de la Señal, Universidad de Alicante, \\ Apartado de correos 99, Alicante E-03080, Spain. \\ bepartamento de Óptica, Farmacología y Anatomía, Universidad de Alicante, Apartado de correos \\ 99, Alicante E-03080, Spain
}

*Corresponding author: $\underline{\text { ramirez@ua.es }}$

\begin{abstract}
The recording of volume holographic reflection gratings in eco-friendly photopolymers represents a challenge at present since they can be used in many important applications such as holographic optical elements and biosensors. In this sense, the aim of this work has been fabricated reflection gratings in the symmetrical experimental in "Biophotopol" and to study the dependence of diffraction efficiency on physical thickness, recording intensity and exposure energy. An increase in diffraction efficiency was observed when the photopolymer films were cured with a LED lamp to improve the stability of the reflection holograms. The maximums diffraction efficiencies around $30 \%$ were obtained for reflection gratings with a spatial frequency of 4888 lines $/ \mathrm{mm}$. The index modulation and optical thickness were obtained by fitting procedure through Kogelnik's coupled wave theory. Experimental and theoretical results have been interpreted to modify the photopolymer formulation and exposure conditions in order to increase the diffraction efficiencies.
\end{abstract}

Keywords: Eco-friendly photopolymers, holographic reflection gratings, LED-curing, volume holography

\section{INTRODUCTION}

Reflection holographic gratings have great interest due to the demand for materials with enough resolution for high spatial frequencies recording. The reflection holograms have been employed for holography display ${ }^{1,2}$, optical data storage and three-dimensional multiplexing to improve the data storage density ${ }^{3,4}$. One of the main advantages of reflection holograms is that they can be reconstructed using white light. This feature has allowed that many investigations have focused on the development of sensors for different types of analytes ${ }^{5-7}$. Thermosensitive reflection holograms have also been investigated ${ }^{8}$.

Today's society demands a scientific development based on green technologies to reduce as low as possible the environmental impact. In this sense, the interest in the development of eco-friendly and low-toxicity photopolymers for holographic recording in reflection mode has reached great relevance in recent years. The majority of hydrophilic photopolymers investigated contain poly(vinyl alcohol) (PVA), gelatin binders or monomers related to acrylamide ${ }^{9-12}$. The last compound is carcinogenic and toxic used in its monomer form. In order to avoid this type of risk for health and environment, we employed a photopolymer developed in our researcher group called Biophotopol as a recording holographic material for optical applications. Biophotopol has low toxicity, good recycling properties and it is environmentally-friendly ${ }^{13-16}$.

The preparation conditions of the prepolymer solutions in our previous studies were controlled to obtain photopolymer layers with the suitable optical properties that provide the highest diffraction efficiencies $(D E)$, i.e. the ratio between the power of diffracted and incident beams. Holographic transmission gratings were stored in Biophotopol in these works ${ }^{13,15}$. Volume phase transmission lenses have also been fabricated in this photopolymer ${ }^{17}$. However, there are not study about reflection grating storing in Biophotopol. The strict control in the preparation conditions of photopolymer layers acquires greater relevance when high spatial frequencies are stored in the material. As spatial frequency increase, the $D E$ becomes lower due to the material cannot resolve correctly the high number of interference fringes. Chain transfer agents (CTA), such as acid citric ${ }^{18}$ or $4,4^{\prime}$-azobis(4-cyanopentanoic acid) ${ }^{19}$, and free radical scavengers (FRS), such as glycerol, can be

Holography: Advances and Modern Trends VI, edited by Antonio Fimia, Miroslav Hrabovský, John T. Sheridan, Proc. of SPIE Vol. 11030, 110300E · () 2019 SPIE · CCC code: 0277-786X/19/\$18 · doi: 10.1117/12.2521439 
incorporated into the photopolymer compositions to improve the response. The mechanics consist of stop the growth of short polymer chains to increase the $D E$. A low-toxicity diacetone acrylamide-based material containing citric acid and glycerol have been reported to store a 3050 lines $/ \mathrm{mm}$ grating in mode reflection with a $D E$ of up to $50 \%$. It is necessary to note that the correct comparison of $D E$ must be made for the same spatial frequencies.

In this work, unslanted holographic reflection gratings were stored in the symmetrical experimental setup in "Biophotopol" at a high spatial frequency of 4738 and 4888 lines $/ \mathrm{mm}$ at recording wavelengths $\left(\lambda_{\text {rec }}\right)$ of 488 and $473 \mathrm{~nm}$. The photopolymer layers uniformity was highly sensitive to drying and environmental conditions during the exposure stage. Therefore, both conditions were investigated. The physical thickness and exposure energy were studied. An increase in diffraction efficiency was observed when the photopolymer films were cured with a LED lamp to improve the stability of the reflection holograms. With the aim to understand the behaviour of the $D E$, the Kogelnik's Coupled wave theory was used to fit the experimental values and to obtain parameters such as the refractive index modulation and the optical thickness. CTAs and FRSs were not used in this research to observe the Biophotopol photopolymer behaviour in reflexion mode.

\section{EXPERIMENTAL PROCEDURE}

\subsection{Material preparation}

Holographic reflection gratings were stored in Biophotopol (the average refractive index is $n \sim 1.5$ ). The prepolymer solution was composed of poly(vinyl alcohol) (PVA) as an inert binder polymer, sodium acrylate (NaAO) as a polymerizable monomer, triethanolamine (TEA) as coinitiator and plasticizer and sodium salt 5 '-riboflavin monophosphate (RF) as sensitizer dye. The solvent used was water, which all components were soluble. The optimized concentrations in the prepolymer solution were $13.0 \mathrm{w} / \mathrm{w} \%, 0.39 \mathrm{M}, 9.0 \times 10^{-3} \mathrm{M}, 1.0 \times 10^{-3} \mathrm{M}$ for PVA, NaAO, TEA and RF, respectively. All compounds were purchased from Sigma-Aldrich Quimica SL (Madrid, Spain).

The prepolymer solution is deposited through the force of gravity on levelled glass plate $(6.3 \times 6.3 \mathrm{~cm})$, which has been previously washed and dried, and left inside an incubator (Climacell 111) with controlled conditions $(60 \pm 5 \%$ relative humidity and $20 \pm 1^{\circ}$ temperature). The process is carried out under controlled light conditions in which the material is not sensitive. The hologram stability has been ensured and increased by a cured process with a LED lamp $(13.5 \mathrm{~W}, 875 \mathrm{~lm}$ at $6500 \mathrm{~K}$, Lexman). In order to determine the optimum drying time for which the highest $D E$ is obtained, the photopolymer layers were dried during several times. A curing time of $20 \mathrm{~min}$ was selected for carried out the curing process of the Biophotopol layers after recording stage. The physical thickness of the photopolymer layers $(h)$ was measured with an ultrasonic pulse-echo gauge (PosiTector 200, DeFelsko, Ogdensburg, NY, USA).

\subsection{Holographic reflection recording}

The experimental holographic set-up used is shown in Figure 1. First, the beam laser is spatially filtered and collimated. Then, this beam is split into two secondary beams, the reference and object beams, using a beam-splitter. The holographic reflection gratings were recorded by the appropriate interference between them. The two laser beams were spatially overlapped at the sample, reaching symmetrically by the opposite sides at the photopolymer layer with a recording angles $\theta_{o}=\theta_{r}=72.9^{\circ}$ with respect to the normal incidence. A continuous $(\mathrm{CW})$ Argon Ion laser tuned at $\lambda_{\text {rec }}=488 \mathrm{~nm}$ and a solid-state laser emitting at $\lambda_{\text {rec }}=473 \mathrm{~nm}$ were used. The material is sensitive for these wavelengths as observed in Figure 2 which the absorption for a $150 \mu \mathrm{m}$ photopolymer layer is shown. The optical density at 488 and $473 \mathrm{~nm}$ are 0.74 and 1.19 , respectively. A greater absorption may be expected to improve the holographic photopolymer properties. The ratio of intensities between both reference and object beams was 1.1. A total recording intensity (sum of both intensity beams measures in the hologram plane) of 13.7 (at $488 \mathrm{~nm}$ ) and $33.2 \mathrm{~mW} / \mathrm{cm}^{2}$ (at $473 \mathrm{~nm}$ ) were used and the exposure times were varied to obtain a range of $H$ from 70.4 to $1660.9 \mathrm{~mJ} / \mathrm{cm}^{2}$.

According to Bragg's law for symmetrical reflection geometry [equation (1)], theoretical spatial frequencies of 4738 and 4888 lines $/ \mathrm{mm}$ were obtained with a $\lambda_{\text {rec }}$ of 488 and $473 \mathrm{~nm}$, respectively.

$$
\Lambda=\frac{\lambda}{2 \sqrt{n^{2}-\sin ^{2} \theta}}
$$




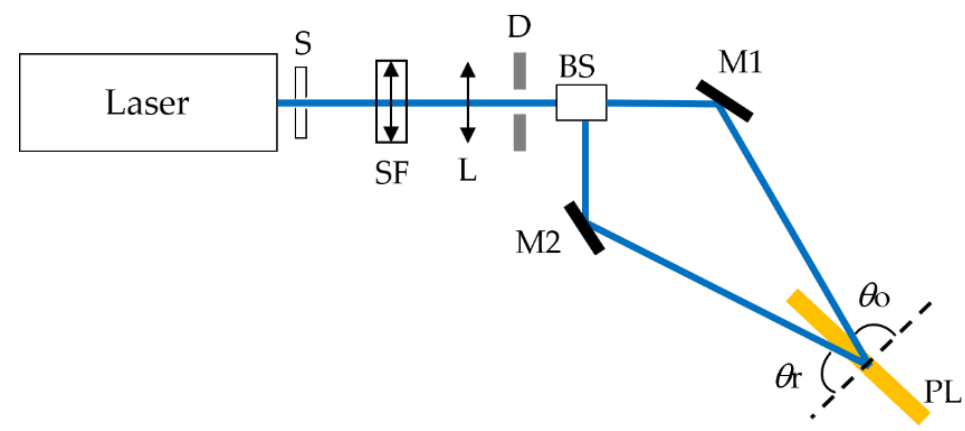

Figure 1. Holographic setup for reflection gratings. BS: beam splitter; SF: spatial filters (microscope objective and pinhole); $\mathrm{M}_{\mathrm{i}}$ : mirrors; L: lens; $\theta_{o}, \theta_{\mathrm{r}}$ : object and reference recording angle; PL: photopolymer layer.

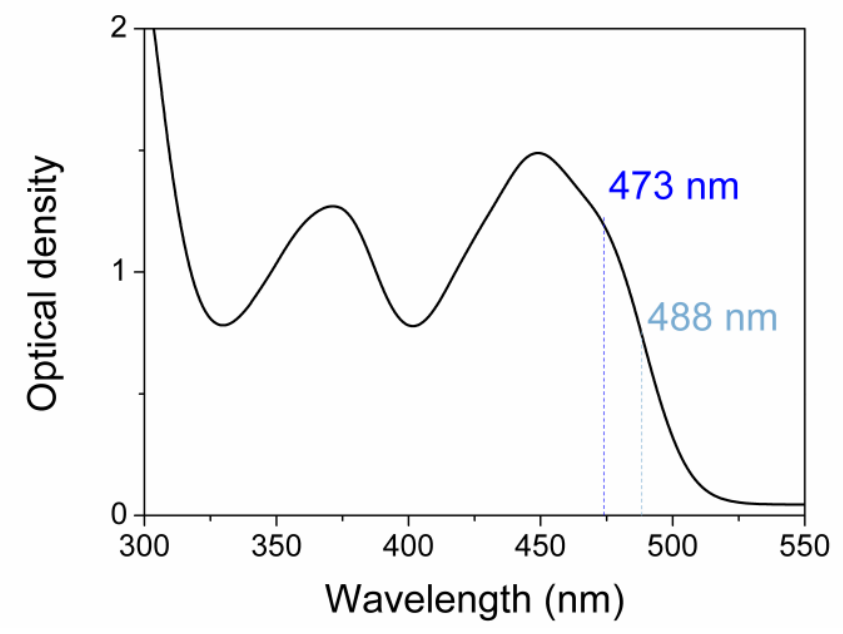

Figure 2. Optical density for $150 \mu \mathrm{m}$ photopolymer layer.

\subsection{Analysis of experimental data through Kogelnik's Coupled Wave Theory}

$D E$ can be obtained as a function of the photopolymer layer transmittance with $\left(T_{\mathrm{pg}}\right)$ and without $\left(T_{\mathrm{p}}\right)$ the stored grating [equation (2)]. The transmission and optical density spectrum at normal incidence upon the photopolymer samples were measured with a double beam spectrophotometer (V-650, Jasco).

$$
D E=\frac{T_{\mathrm{p}}-T_{\mathrm{pg}}}{T_{\mathrm{p}}}
$$

According to Kogelnik's Coupled wave theory ${ }^{20}$, the DE of phase holographic reflection gratings is given by:

$$
D E=e^{\left(-\alpha d^{\prime} / \cos \theta^{\prime}\right)}\left(1+\frac{1-\frac{\xi^{2}}{v^{2}}}{\sinh ^{2} \sqrt{v^{2}-\xi^{2}}}\right)^{-1}
$$


The experimental transmission values as a function of the wavelength can be fitted by means of efficient transmission $(T E)$ relation of Kogelnik's Coupled wave theory [equation (4)]. The optical thickness $(d), \Delta n, \Lambda, n$, absorption loss coefficient $(\alpha)$ and fringe tip angles $(\varphi)$ were obtained.

$$
T E=e^{\left(-\alpha d^{\prime} / \cos \theta^{\prime}\right)}\left[1-\left(1+\frac{1-\frac{\xi^{2}}{v^{2}}}{\sinh ^{2} \sqrt{v^{2}-\xi^{2}}}\right)^{-1}\right]
$$

The parameter that controls $D E$ at Bragg condition is $v$ [Equation (6)], while the $\xi$ parameter [equation (6)] is related to the deviation from the exact Bragg condition.

$$
\begin{gathered}
v=i \frac{\pi \Delta n d^{\prime}}{\lambda \sqrt{c_{r} c_{S}}} \\
\xi=-\frac{\pi d^{\prime}}{\Lambda c_{S}}\left[\left|\sin \left(\theta^{\prime}-\varphi\right)\right|-\frac{\lambda}{2 n \Lambda}\right]
\end{gathered}
$$

Slant factors $\left(c_{\mathrm{r}}\right.$ and $\left.c_{\mathrm{s}}\right)$ are defined in equation (7) and equation (8), respectively.

$$
\begin{gathered}
c_{r}=\cos \theta^{\prime} \\
c_{S}=\cos \theta^{\prime}-\frac{\lambda}{n \Lambda} \sin \varphi
\end{gathered}
$$

\section{RESULTS AND DISCUSSION}

The weight of the photopolymer layers was monitored as a function of the time that the films remain inside of incubator with controlled humidity $(60 \%)$ and temperature $\left(20^{\circ} \mathrm{C}\right)$ with the aim to determinate the optimal drying time in which the higher $D E_{\max }$ is obtained. Figure 2 shows the measures when an initial amount of $3.03 \mathrm{~g}$ prepolymer solution is deposited over a glass plate and introduced in the incubator. Three zones with different drying speeds can be clearly observed. In zone I, a loss of $47.5 \%$ respect to the initial weight of the photopolymer layer occurs during the first 9.7 hours of drying. From this time, the drying speed decreases (zone II). The weight measured decrease very slightly and remains practically constant from 21.4 hours until the measurement is finished. It is denoted in Figure 2 as zone III. The $D E_{\max }$ was measured in the three zones. The $D E_{\max }$ in the zones I and II are practically zero while the maximum $D E_{\max }$ value is obtained when the zone II begins at $21.4 \mathrm{~h}$ of drying. This time value is selected as the optimal drying time. Identical experiments were realized for different amounts of prepolymer solution deposited over the glass plates and the optimal drying time was obtained for the different physical thickness of the photopolymer layers. 


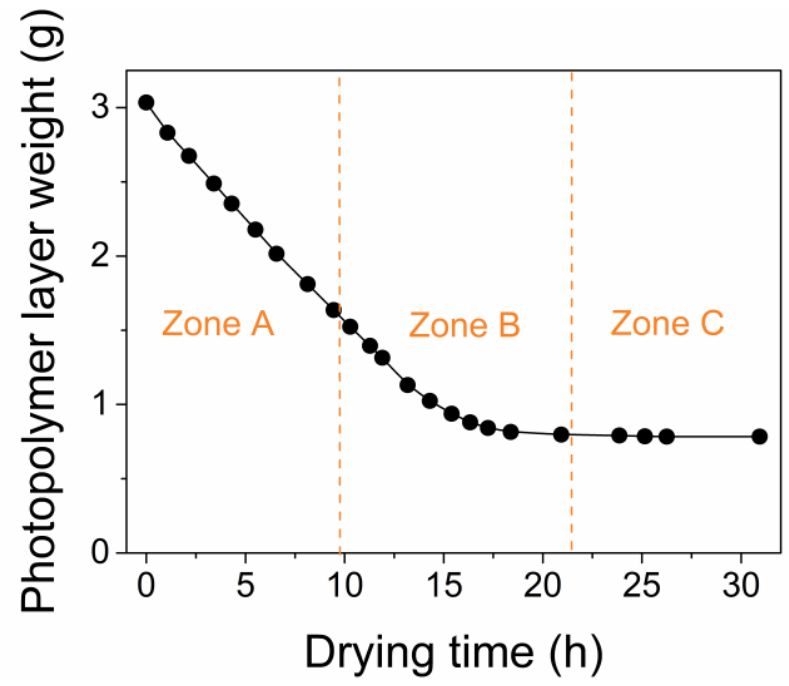

Figure 2. Weight loss (black circles) of a photopolymer layer when an initial amount of $3.03 \mathrm{~g}$ prepolymer solution is deposited over a glass plate.

Once the optimal drying times are determined and in order to determine the optimum physical thickness for which the highest $D E_{\max }$ is obtained, the thicknesses of the dried layers in the range $80-200 \mu \mathrm{m}$ were investigated. Figure 3 shows the normalized $D E_{\max }$ for these thicknesses. As clearly can be observed, the maximum $D E_{\max }$ is reaching when the $h=145$ $\mu \mathrm{m}$. From this value, the $D E_{\max }$ decrease due possibly to the greater attenuation of the beams when penetrating in the layer. Also, and considering that the interference fringes are formed from the centre of the photopolymer layer outwards, both beams must be subjected to the same material attenuation and thus, the interferences would take place correctly. However, a gradient concentration of dye molecules between both sides of the sample could exist, causing an unequal attenuation between them.

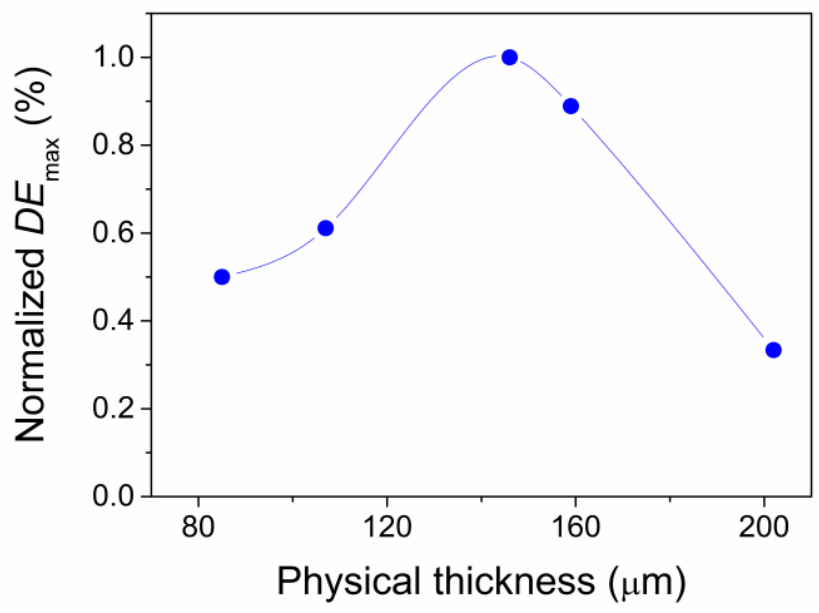

Figure 3. Normalized $D E_{\max }$ vs physical thickness of photopolymer layers. $H=320.0 \mathrm{~mJ} / \mathrm{cm}^{2}$ was used in the recording stage. 
The influence of $H$ on the $D E_{\max }$ after both recording and curing stages (RS and CS, respectively) was investigated. Biophotopol layers with a physical thickness of $145 \mu \mathrm{m}$ were used for recording reflection gratings with spatial frequencies of 4738 and 4888 lines $/ \mathrm{mm}$. A theoretical analysis of the holographic grating parameters was realized to understand the $D E_{\max }$ behaviour between both stages. The optical parameters were obtained through the theoretical fit of the experimental data with the Kogelnik's Coupled wave theory [equation (4)]. Figure 5 shows the experimental transmittance spectra measured immediately after recording (black squares) and curing stage (orange circles) for a reflection grating recorded with a total $H$ of $70.4,320.0$ and $1660.9 \mathrm{~mJ} / \mathrm{cm}^{2}$. The $D E_{\max }$ is calculated from the experimental data by means of equation (2) and the theoretical fits are represented with solid lines. The results are presented in Table 1.
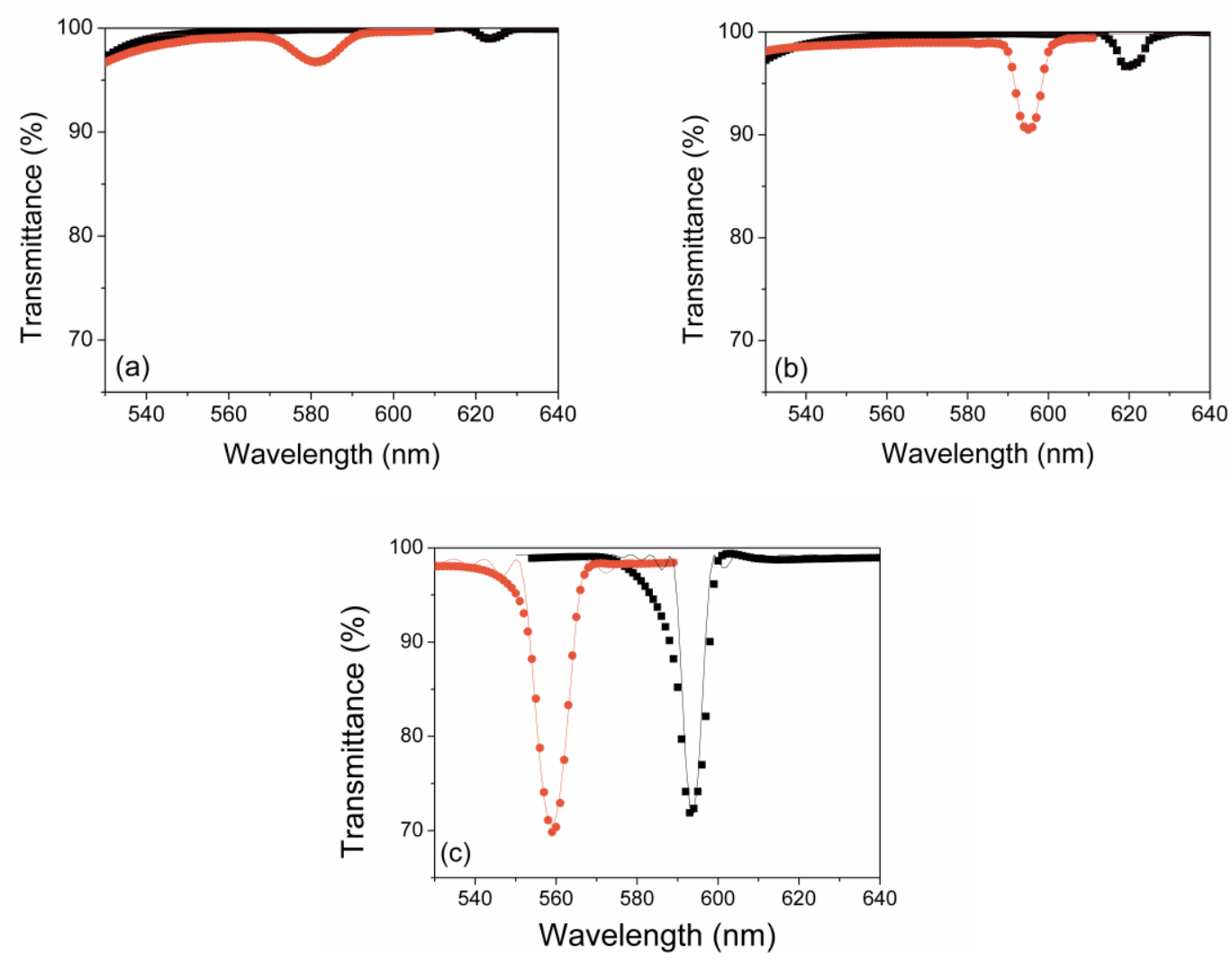

Figure 4. Transmittance of reflection gratings recorded at 70.4 (a), 320.0 (b) and 1660.9 (c) $\mathrm{mJ} / \mathrm{cm}^{2}$ for recording (black squares) and curing (orange circles) stages as a function of reconstruction wavelength. The spatial frequency of the gratings were 4738 [(a) and (b)] and 4888 (c) lines/mm and $h=145 \mu \mathrm{m}$. Solid lines represent the theoretical fit through Kogelnik's Coupled wave theory.

In all measures, $D E_{\max }$ in CS is greater than those obtained in RS. The maximum $D E_{\max }$ reached was $31.3 \%$ at $H=1660.9$ $\mathrm{mJ} / \mathrm{cm}^{2}$ and $\lambda_{\text {rec }}=473 \mathrm{~nm}$. The $\Delta n$ is one of the most important optical parameters in the $D E$ of a holographic grating. $\Delta n$ for RS $\left(\Delta n_{\mathrm{RS}}\right)$ is smaller than $\Delta n$ for CS $\left(\Delta n_{\mathrm{CS}}\right)$ in the $H$ range investigated. The maximum values of $\Delta n_{\mathrm{RS}}(0.0050)$ and $\Delta n_{\mathrm{CS}}(0.0091)$ are obtained at $1660.9 \mathrm{~mJ} / \mathrm{cm}^{2}$ when a $\lambda_{\text {rec }}=473 \mathrm{~nm}$ is used. Regarding optical thickness, $\left(d_{\mathrm{RS}}\right.$ and $d_{\mathrm{CS}}$ denoted the optical thickness obtained after the recording and curing stage, respectively), when the curing stage is carried out, $d_{\mathrm{CS}}$ decrease respect to $d_{\mathrm{RS}}$ values. This fact can be explained due to a dimensional change in the material that can occur during the curing process. The total conversion of the monomer molecules into a polymer network causes a volume reduction produced by the close packing between polymer chains. This is known as photopolymerization shrinkage. It is interesting to note that this phenomenon is very important in the case of reflection gratings due to the high number of interference fringes. From equation (4), the maximum $D E_{\max }$ is obtained at the Bragg condition (in which the parameter $\xi$ is 0 ). In this point, $D E$ is dominated by parameter $v$, which depends on the product $\Delta n \cdot d$. As observed in Table 1 , this 
product is one order of magnitude higher for $\lambda_{\text {rec }}=473 \mathrm{~nm}$ in comparison with $\lambda_{\text {rec }}=488 \mathrm{~nm}$ in both RS and CS. $\Delta n \cdot d$ obtained in CS are greater than $\Delta n \cdot d$ reached in RS for a recording wavelength of $488 \mathrm{~nm}$. However, for $\lambda_{\text {rec }}=473 \mathrm{~nm}$, $\Delta n \cdot d$ in CS is practically the same as $\Delta n \cdot d$ obtained in RS. This behaviour means that the $D E_{\max }$ are very similar.

Table 1. Parameters obtained from the theoretical fit of the experimental transmittance values.

\begin{tabular}{|c|c|c|c|c|c|c|}
\hline$\lambda_{\text {rec }}(\boldsymbol{\mu m})$ & $\boldsymbol{H}\left(\mathbf{m} \mathbf{J} / \mathbf{c m}^{2}\right)$ & Stage & $\boldsymbol{\Delta n}$ & $\boldsymbol{d}(\boldsymbol{\mu m})$ & $\boldsymbol{\Delta} \boldsymbol{n} \cdot \boldsymbol{d}(\boldsymbol{\mu m})$ & $\boldsymbol{D} \boldsymbol{E}_{\max }(\boldsymbol{\%})$ \\
\hline 0.488 & 70.4 & RS & 0.0012 & 16.0 & 0.019 & 0.9 \\
& & CS & 0.0035 & 8.6 & 0.030 & 2.7 \\
\hline 0.488 & 320.0 & RS & 0.0018 & 20.7 & 0.037 & 3.6 \\
& & CS & 0.0036 & 16.5 & 0.059 & 9.5 \\
\hline 0.473 & 1660.9 & RS & 0.0050 & 22.2 & 0.111 & 29.7 \\
& & CS & 0.0091 & 12.3 & 0.112 & 31.3 \\
\hline
\end{tabular}

\section{CONCLUSIONS}

In this work, reflection holographic gratings in an environmentally-friendly photopolymer prepared at optimum conditions have been shown. A curing process with a low-cost LED lamp increases the $D E_{\max }$ achieved in the recording stage and stabilizes the hologram behaviour over time. The $D E_{\max }$ of $31.3 \%$ obtained with these gratings is one of the best values published in reflection holographic gratings over green photopolymers.

\section{ACKNOWLEDGEMENTS}

Ministerio de Ciencia, Innovación y Universidades, Spain, under projects FIS2017-82919-R (MINECO/AEI/FEDER, UE) and FIS2015-66570-P (MINECO/FEDER) and by Universidad de Alicante under project GRE17-06.

\section{REFERENCES}

[1] Bjelkhagen, H. I. and Mirlis, E., "Color holography to produce highly realistic three-dimensional images.," Appl. Opt. 47(4), A123-33 (2008).

[2] Defosse, Y., Carre, C. and Lougnot, D. J., "Use of a self-developing polymer material for volume reflection hologram recording," Pure Appl. Opt. J. Eur. Opt. Soc. Part A 2(5), 437-440 (1993).

[3] Fuentes, R., Fernández, E., García, C., Beléndez, A. and Pascual, I., "Study of reflection gratings recorded in polyvinyl alcohol/acrylamide-based photopolymer," Appl. Opt. 48(34), 6553-6557 (2009).

[4] Yonetani, Y., Nitta, K. and Matoba, O., "Numerical evaluation of angular multiplexing in reflection-type holographic data storage in photopolymer with shrinkage.," Appl. Opt. 49(4), 694-700 (2010).

[5] Yetisen, A. K., Montelongo, Y., Qasim, M. M., Butt, H., Wilkinson, T. D., Monteiro, M. J. and Yun, S. H., "Photonic nanosensor for colorimetric detection of metal ions," Anal. Chem. 87(10), 5101-5108 (2015).

[6] Liu, H., Wang, R., Yu, D., Luo, S., Li, L., Wang, W. and Song, Q., "Direct light written holographic volume grating as a novel optical platform for sensing characterization of solution," Opt. Laser Technol. 109, 510-517 (2019).

[7] Liu, H., Yu, D., Zhou, K., Wang, S., Luo, S., Li, L., Wang, W. and Song, Q., "Novel pH-sensitive photopolymer hydrogel and its holographic sensing response for solution characterization," Opt. Laser Technol. 101, 257-267 (2018).

[8] Mikulchyk, T., Martin, S. and Naydenova, I., "N-isopropylacrylamide-based photopolymer for holographic 
recording of thermosensitive transmission and reflection gratings," Appl. Opt. 56(22), 6348 (2017).

[9] Gallego, S., Ortuño, M., Neipp, C., Márquez, A., Beléndez, A. and Pascual, I., "Characterization of polyvinyl alcohol/acrylamide holographic memories with a first-harmonic diffusion model," Appl. Opt. 44(29), 6205-6210 (2005).

[10] Gallego, S., Márquez, A., Méndez, D., Neipp, C., Ortuño, M., Beléndez, A., Fernández, E. and Pascual, I., "Direct analysis of monomer diffusion times in polyvinyl/acrylamide materials," Appl. Phys. Lett. 92(7), 073306 (2008).

[11] Weiss, V., Millul, E. and Friesem, A. A., "Photopolymeric holographic recording media: in-situ and real-time characterization," Proc. SPIE 2688, 11-21 (1996).

[12] Ortuño, M., Gallego, S., García, C., Pascual, I., Neipp, C. and Beléndez, A., "Holographic Characteristics of an Acrylamide/Bisacrylamide Photopolymer in 40-1000 ?m Thick Layers," Phys. Scr., 66 (2005).

[13] Ortuño, M., Gallego, S., Márquez, A., Neipp, C., Pascual, I. and Beléndez, A., "Biophotopol: A Sustainable Photopolymer for Holographic Data Storage Applications," Materials (Basel). 5(12), 772-783 (2012).

[14] Navarro-Fuster, V., Ortuño, M., Fernández, R., Gallego, S., Marquez, A., Beléndez, A. and Pascual, I., "Peristrophic multiplexed holograms recorded in a low toxicity photopolymer," Opt. Mater. Express 7(1), 133147 (2017).

[15] Navarro-Fuster, V., Ortuño, M., Gallego, S., Márquez, A., Beléndez, A. and Pascual, I., "Biophotopol's energetic sensitivity improved in $300 \mu \mathrm{m}$ layers by tuning the recording wavelength," Opt. Mater. (Amst). 52, 111-115 (2016).

[16] Ortuño, M., Fernández, E., Gallego, S., Beléndez, A. and Pascual, I., "New photopolymer holographic recording material with sustainable design.," Opt. Express 15(19), 12425-12435 (2007).

[17] Lloret, T. ., Navarro-Fuster, V. ., Ramírez, M. G. ., Ortuño, M. ., Neipp, C., Beléndez, A. . and Pascual, I. ., "Holographic Lenses in an Environment-Friendly Photopolymer," Polymers (Basel). 10(3), 302 (2018).

[18] Cody, D., Gribbin, S., Mihaylova, E. and Naydenova, I., "Low-Toxicity Photopolymer for Reflection Holography," ACS Appl. Mater. Interfaces 8(28), 18481-18487 (2016).

[19] Fernandez, E., Fuentes, R., Belendez, A. and Pascual, I., "Influence of 4,4'-azobis (4-cyanopentanoic acid) in Transmission and Reflection Gratings Stored in a PVA/AA Photopolymer," Materials (Basel). 9(3), 194 (2016).

[20] Kogelnik, H., “Coupled Wave Theory for Thick Hologram Gratings,” Bell Syst. Tech. J. 48(9), 2909-2947 (1969). 